\title{
Asymmetrically cut crystals as optical elements for highly collimated x-ray beams
}

S. Brauer and G. B. Stephenson

IBM Research Division, Thomas J. Watson Research Center, Yorktown Heights, New York 10598

M. Sutton

Department of Physics, McGill University, Montréal, Québec H3A 2T8, Canada

S. G. J. Mochrie

Department of Physics, Massachusetts Institute of Technology, Cambridge, Massachusetts 02139

S. B. Dierker

Department of Physics, University of Michigan, Ann Arbor, Michigan 48109

R. M. Fleming and R. Pindak

AT\&T Bell Laboratories, Murray Hill, New Jersey 07974

I. K. Robinson

Department of Physics, University of Illinois, Urbana, Illinois 61801

G. Grübel, J. Als-Nielsen, and D. L. Abernathy

European Synchrotron Radiation Facility, BP 220, F-38043 Grenoble Cedex, France

(Presented on 18 July 1994)

Asymmetrically cut perfect crystals, in both the Laue and Bragg geometries, are examined as single crystal monochromators for $\mathrm{x}$-ray beams that are collimated to a small fraction of the Darwin width, as is typical in experiments with coherent $\mathrm{x}$ rays. Both the Laue and asymmetric Bragg geometries are plagued by an inherent chromatic aberration that increases the beam divergence much beyond that of the symmetric Bragg geometry. Measurements from a recent experiment at the ESRF are presented to compare $\mathrm{Si}(220)$ (symmetric Bragg), diamond(111) (asymmetric Laue), and diamond(111) (symmetric Bragg inclined) geometries. (O) 1995 American Institute of Physics.

\section{INTRODUCTION}

Unlike most previous $\mathrm{x}$-ray sources, undulators provide a beam with a collimation which is smaller than the angular acceptance (Darwin width) of a typical monochromator crystal. To devise crystal optics for these sources, it is necessary to understand the detailed optical behavior of diffracting crystals, such as the exact angles the exit beam makes with the incident beam, on a scale finer than the Darwin width. Understanding these properties is particularly important in the new field of coherent $\mathrm{x}$-ray science, where the $\mathrm{x}$-ray beams are further collimated to a small fraction of the total source divergence.

In this paper, we describe the optical properties of both Bragg and Laue geometries, particularly the transmitted wavelength range and exit divergence, for incident radiation which is polychromatic and highly collimated on the scale of the Darwin width. We then present experimental results for crystals in various geometries, including single-crystal transmission monochromators.

\section{PROPERTIES OF ASYMMETRICALLY CUT CRYSTALS}

Asymmetric Bragg and Laue crystal geometries are shown in Fig. 1, where $\alpha$ is the angle between the crystal surface and the lattice planes. Just as in the symmetric Bragg case $(\alpha=0)$, when the diffraction conditions are met, the angles between the reflecting lattice planes and both the incident and exit beams are approximately equal to the Bragg angles $\theta_{B}$. For a crystal of lattice spacing $d$, the Bragg angle for wavelength $\lambda$ is given by $\theta_{B} \equiv \sin ^{-1}(\lambda / 2 d)$. The exact angles of the incident and exit beams with respect to the surface of the crystal may be expressed as

$$
\theta_{i}=\theta_{B}+\alpha+\Delta \theta_{i} \quad \text { and } \theta_{e}=\theta_{B}-\alpha+\Delta \theta_{e},
$$

respectively, where $\Delta \theta_{i}$ and $\Delta \theta_{e}$ are small $(<100 \mu \mathrm{rad})$ deviations of the incident and exit beam directions from the Bragg-law directions. These deviations arise because of both the finite width of the reflection (Darwin width) and a correction arising from the index of refraction within the crystal. For a perfect crystal with a flat surface, there is an exact one-to-one relationship between $\theta_{i}$ and $\theta_{e}$ for a given wavelength. Thus, although the reflectivity is near unity over a range of incidence angles, a perfectly collimated and monochromatic beam incident within this range will produce a diffracted beam at a single exit angle.

The asymmetry of the surface orientation can be characterized by an asymmetry factor $b$, which is conventionally 1 written as

$$
b \equiv \frac{\sin \left(\alpha+\theta_{B}\right)}{\sin \left(\alpha-\theta_{B}\right)} .
$$

A given crystal has fixed values of $\alpha$ and $d$, and the parameter $b$ depends implicitly on wavelength through $\theta_{B}$. For Bragg geometries we have $\alpha<\theta_{B}$ so that $b<0$ and $\theta_{c}>0$ (reflection). In Laue geometries, we have $\alpha>\theta_{B}$ so that $b>0$ 


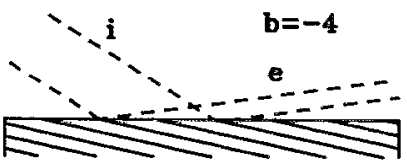

(a)

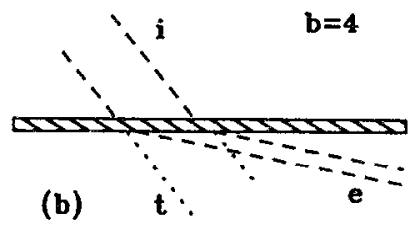

(b)
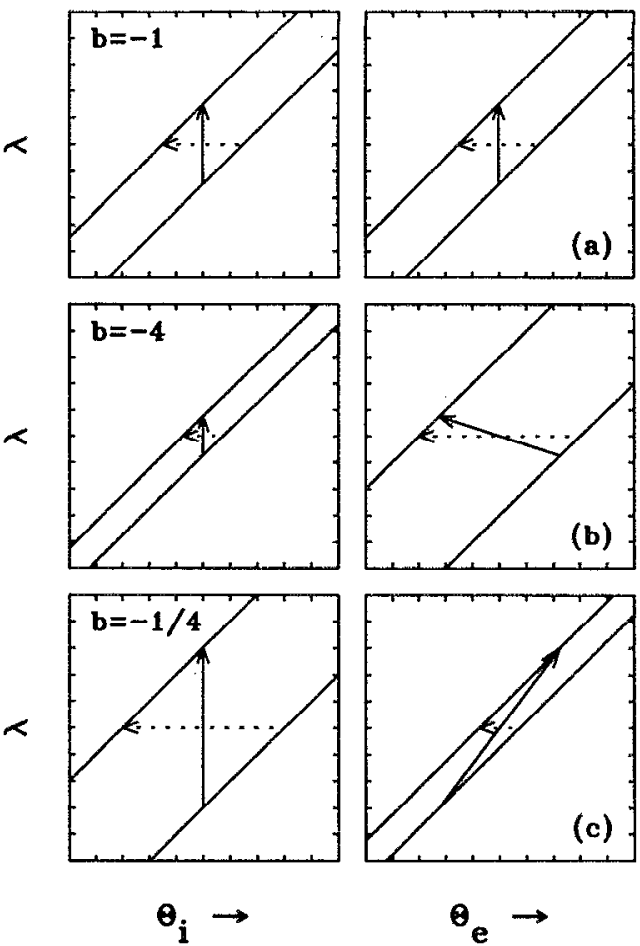

$\theta_{\mathrm{e}} \rightarrow$
The precise shape of the reflectivity as a function of
FIG. 1. Two asymmetric crystal geometries with the same Bragg angle $\theta_{B}=20^{\circ}$. A miscut angle of $\alpha=12.32$ gives a Bragg geometry with $b=-4$ (a) while a miscut of $\alpha=31.25$ gives a Laue geometry with $b=4$ (b). The dashed lines indicate the incident $(i)$ and exit $(e)$ beams while the dotted lines represent the forward diffracted and transmitted beams $(t)$.

and $\theta_{e}<0$ (transmission). The value $b=-1$ represents the symmetric Bragg case $(\alpha=0)$, while $b=1$ represents the symmetric Laue casc $(\alpha=\pi / 2)$. incidence angle at fixed wavelength differs between the Bragg and Laue geometries. However, when the effects of absorption can be neglected, the angular width of the reflectivity has the same magnitude in both geometries, for the same magnitude of asymmetry $|b|$. The half-widths on the incidence and exit side, $w_{i}$ and $w_{e}$, respectively, are related $^{1,2}$ in a manner which varies with asymmetry according to

$$
w_{e}=-b w_{i}=-w_{s}|b|^{1 / 2},
$$

where $w_{s}$ is the magnitude of the half-width for symmetric reflection. Likewise, the one-to-one relationship between incidence and exit angle at a particular wavelength is given by

$$
\Delta \theta_{e}=-b \Delta \theta_{i} \text {. }
$$

These relations hold for both Bragg and Laue geometries. Note that the signs of $\Delta \theta_{i}$ and $\Delta \theta_{e}$ (and $w_{i}$ and $w_{e}$ ) are the same for Bragg geometries but differ for Laue.

\section{A. DuMond diagrams}

To understand the performance of crystal optics for synchrotron studies, one must generally account for the polychromatic nature of the radiation. The relationship between incidence and exit angles, for each wavelength, can be conveniently illustrated in a manner put forward by DuMond. ${ }^{3}$ For example, the DuMond diagram shown in Fig. 2(a) shows the wavelength as a function of incidence angle on one side and exit angle on the other, for the symmetric Bragg geometry. The region between the two diagonal lines on the incidence side represents the range wavelengths and angles which could be accepted for diffraction by the crystal. (We only depict the narrow region near the diffraction condition, where the diagonal lines have a slope of $\cos \theta_{B}$.) We will refer to this region as the reflectivity band. The analogous reflectivity band on the exit side represents the range of possible wavelengths and angles in the exit beam. As described by Eq. (4), each point on the incident side maps onto one point on the exit side, with the wavelength being unchanged.

We discuss the optical performance of a variety of crystal geometries in the subsequent sections. Each geometry will be considered under three different conditions of incident illumination: (1) perfectly monochromatic, uncollimated, (2) polychromatic, perfectly collimated, and (3) poly-
FIG. 2. DuMond diagrams for Bragg geometries with (a) $b=-1$, (b) $b=$ -4 , and (c) $b=-1 / 4$. Radiation accepted from perfectly monochromatic uncollimated incident radiation is shown with dashed arrows while that accepted from polychromatic and perfectly collimated incident radiation is shown with solid arrows.

chromatic with a finite divergence. The second case is a good approximation for experiments with coherent $\mathrm{x}$-ray beams while the lattice case is of more general interest for synchrotron work.

\section{B. Bragg geometries}

Figure 2(a) is a DuMond diagram for the symmetric Bragg geometry. The horizontal arrow on the incidence side represents the range of angles accepted by the crystal under uncollimated monochromatic illumination, i.e., the incidence Darwin width $2\left|w_{i}\right|$. The arrow maps onto an identical arrow on the exit side and the exit divergence $D_{c}$ is equal to the accepted incident divergence $D_{i}$. The vertical arrow on the incidence side represents the accepted range of wavelength $\Delta \lambda$ under perfectly collimated polychromatic illumination. This arrow also maps onto an identical arrow on the exit side indicating that the collimation has been preserved, a property unique to the symmetric Bragg geometry.

Figure 2(b) depicts a Bragg geometry with an asymmetry factor of $b=-4$. By Eqs. (3) we see that the diffracted range of angles for monochromatic radiation (horizontal arrows) is smaller on the incidence side and larger on the exit side relative to the symmetric case, both by a factor of $|b|^{1 / 2}$. Also, if the divergence of the incident monochromatic beam $D_{i}$ only partially fills the incident Darwin width, the divergence of the exit beam is $D_{e}=|b| D_{i}$. The divergence increase is matched by a decrease in the spatial extent of the 

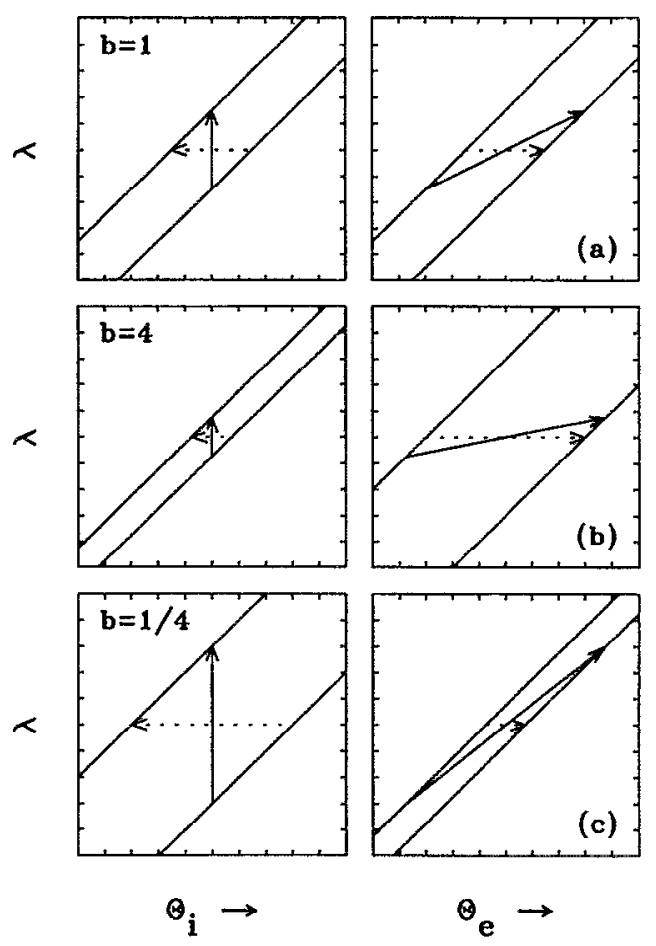

$\theta$

FIG. 3. DuMond diagrams for Laue geometries with (a) $b=1$, (b) $b=4$, and (c) $b=1 / 4$.

beam [shown in Fig. 1(a)] such that the brilliance of a monochromatic beam is preserved.

Strikingly different behavior occurs in the asymmetric Bragg geometry if the incident radiation is perfectly collimated yet polychromatic. As shown by the short vertical arrow on the incidence side, the crystal accepts a wavelength range which is only $|b|^{-1 / 2}$ times as large as a symmetric Bragg crystal. In mapping this arrow onto the exit side the wavelength range is dispersed across an exit angular range of size $2\left|w_{i}-w_{e}\right|$, which is most of the exit Darwin width. This behavior is a form of chromatic aberration which is generally undesirable for experiments involving coherent $x$ rays, where a divergence much narrower than the Darwin width must be preserved.

The DuMond diagram for an asymmetric Bragg crystal with $b=-1 / 4$ is shown in Fig. 2(c). Compared to the symmetric Bragg case, the incidence Darwin width is greater and the exit Darwin width is smaller by the factor $|b|^{-1 / 2}$. With monochromatic radiation, the exit beam is $|b|$ times more collimated than that accepted at the incident side. (Not shown on the diagram is that the exit beam is spatially expanded in proportion to this collimation increase.) For polychromatic collimated radiation, as shown by the vertical arrow on the incidence side, the accepted $\Delta \lambda$ is larger by a factor of $|b|^{-1 / 2}$ and the exit radiation is again dispersed across a wide angular range $2\left|w_{i}-w_{e}\right|$.

These results may be generalized to the case of a polychromatic source with a finite angular divergence. For an incident divergence $D_{i}$, the full ranges of wavelengths and exit angles are given by

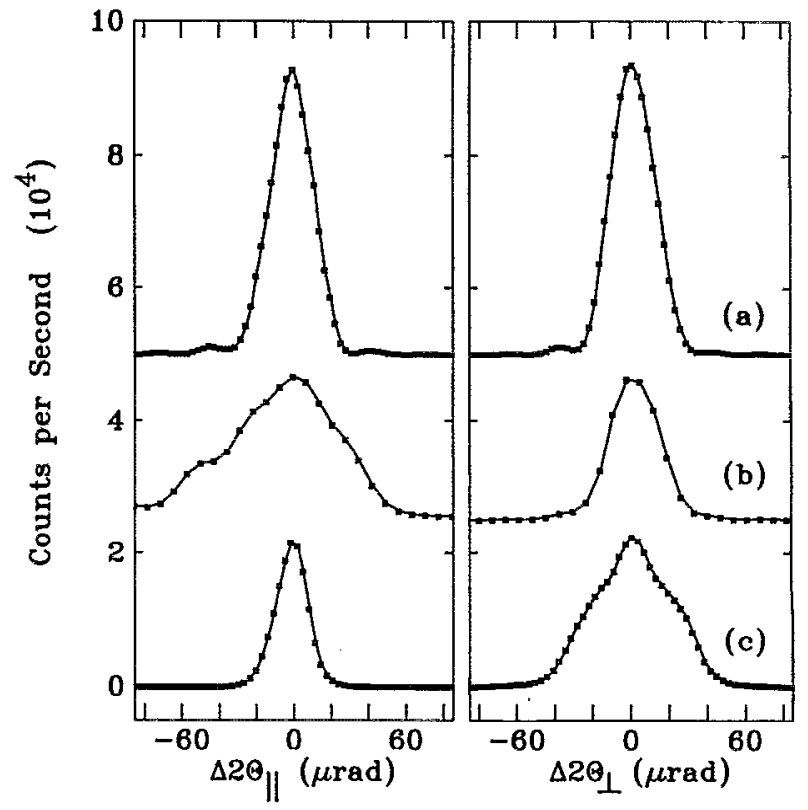

FIG. 4. Angular divergence measurements for $\lambda=1.05 \AA$ radiation passing through a $4 \mu \mathrm{m}$ diameter pinhole aperture located downstream from (a) $\mathrm{Si}(220)$ (symmetric Bragg), (b) $250 \mu \mathrm{m}$ thick diamond(111) (asymmetric Laue), and (c) Si(220) (symmetric Bragg) plus diamond(111) (symmetric Bragg inclined) crystals.

$$
\begin{aligned}
& \frac{\Delta \lambda}{\lambda}=\frac{2\left|w_{i}\right|+D_{i}}{\tan \theta_{B}}, \\
& D_{e}=2\left|w_{i}\right||1+b|+D_{i} .
\end{aligned}
$$

These relations also describe the Laue geometry where $b>0$, as we will now discuss.

\section{Laue geometries}

For Bragg geometries, an incident ray accepted on the low $\theta_{i}$ side of the reflectivity band, will exit the crystal on the low $\theta_{e}$ side. In contrast, the same ray incident on a Laue crystal will exit on the high $\theta_{e}$ side. This difference is clear from Fig. 3(a) which shows the DuMond diagram of a symmetric Laue crystal $(b=1)$. The horizontal arrow representing monochromatic radiation accepted on the incident side, now maps onto an arrow of the same length but opposite direction on the exit side.

Contrast between symmetric Bragg and Laue geometries is more pronounced in the case of polychromatic collimated incident radiation. Here, the vertical arrow on the incident side maps onto a diagonal arrow on the exit side, such that the exit divergence is $2\left|w_{e}\right|$, even though the incident radiation was perfectly collimated.

Figures 3(a)-3(c) for the Laue geometry show that, as for the Bragg geometry, the ratio of $D_{e} / D_{i}$ is equal to $|b|$ for monochromatic light. According to Eq. (5), for polychromatic light $\Delta \lambda$ varies as $|b|^{-1 / 2}$ for small $D_{i}$, as is true for Bragg crystals. The dispersion of wavelengths into angles is stronger for Laue geometries, as is clear from Eq. (6) and Figs. 3(a)-3(c).

In summary, when a Laue or asymmetrically cut Bragg crystal is used with a highly collimated polychromatic $\mathrm{x}$-ray 
beam, the exit divergence is increased much beyond that of the symmetric Bragg case. This results from an inherent chromatic aberration which introduces a strong correlation between angle and wavelength. Used as single-crystal monochromator, such a crystal would degrade the brilliance of the polychromatic beam. However, the degradation is perfectly defined and the brilliance could be regained by using a second crystal of opposite asymmetry.

\section{MEASUREMENTS FROM VARIOUS GEOMETRIES}

At ESRF undulator beamline 9 ('lroika) we have been studying reflection and transmission single-crystal monochromators for experiments requiring highly collimated coherent $x$-ray beams. Figure 4 shows measurements of the exit beam divergence produced by various monochromator crystals. These angular distributions were obtained at $\lambda=1.05 \AA$ by placing a $4 \mu \mathrm{m}$ diameter pinhole in the exit beam and scanning a second pinhole in front of the detector approximately $1 \mathrm{~m}$ downstream. The directions $2 \theta_{\|}$and $2 \theta_{\perp}$ are within and normal to the scattering plane, respectively. The top pair of curves was obtained for a $\mathrm{Si}(220)$ crystal in the symmetric Bragg geometry. The observed $27 \mu \mathrm{rad}$ FWHM in each direction is equal to the width expected for Fraunhofer diffraction from the pinhole combined with instrumental resolution. Within the measurement precision, the crystal does not introduce any extra divergence to the beam.

The middle data are for a diamond crystal with (100) surface normal, oriented for (111) diffraction in an asymmetric Laue configuration $(b=1.46)$. The $2 \theta_{\perp}$ FWHM is 36 $\mu \mathrm{rad}$, which is slightly greater than the width expected from Fraunhofer diffraction plus the (slightly broader) instrumental resolution $(31 \mu \mathrm{rad})$. However, the $2 \theta_{\|}$width $(61 \mu \mathrm{rad})$ greatly exceeds the $2 \theta_{\perp}$, width. The $2 \theta_{\|}$broadening is consistent with that expected from chromatic aberration in this geometry which, according to Eq. (6), introduces an additional divergence of $2\left|w_{i}\right||1+b|=28 \mu \mathrm{rad}$. Crystal mosaic in the diamond will also tend to broaden the widths contributing more in the $2 \theta_{\|}$direction than the $2 \theta_{\perp}$.

Until this point we have been discussing noninclined geometries. The final data in Fig. 4 are for a diamond in the symmetric Bragg inclined geometry. The (100) surfacenormal crystal was oriented for (111) diffraction. [For this measurement a symmetric Bragg $\mathrm{Si}(220)$ crystal was upstream of the inclined crystal but because it has a slightly broader Darwin width it should not affect the measured exit divergence.] The resolution-limited Fraunhofer width is observed in the $2 \theta_{\|}$direction. Now however, the $2 \theta_{\perp}$ intensity distribution is broadened, although it retains some signature of the narrow Fraunhofer peak. We believe this is due to a chromatic aberration analogous to that described above. The broadening is in the direction of $2 \theta_{1}$ rather than $2 \theta_{\|}$because in this geometry the difference between the scattering vector and the surface normal lies in the direction of $2 \theta_{\perp}$ rather than $2 \theta_{\|}$.

${ }^{1}$ B. W. Batterman and H. Cole, Rev. Mod. Phys. 36, 681 (1964).

${ }^{2}$ S. Brauer, G. B. Stephenson, and M. Sutton, J. Appl. Crystallogr. (submitted).

${ }^{3}$ J. W. M. DuMond, Phys. Rev. 52, 872 (1937). 